ENCYCLOPÉDIE Encyclopédie berbère

BERBERE

32 | 2010

32 | Mgild - Mzab

\title{
Mzab (ou M’zab) : Histoire et société
}

S. Doumane

\section{OpenEdition}

Journals

Édition électronique

URL : https://journals.openedition.org/encyclopedieberbere/676

DOI : 10.4000/encyclopedieberbere.676

ISSN : 2262-7197

\section{Éditeur}

Peeters Publishers

\section{Édition imprimée}

Date de publication : 31 décembre 2010

Pagination : $5173-5181$

ISBN : 978-90-429-2369-0

ISSN : 1015-7344

\section{Référence électronique}

S. Doumane, «Mzab (ou M'zab) : Histoire et société », Encyclopédie berbère [En ligne], 32 | 2010, document M153, mis en ligne le 11 novembre 2020, consulté le 17 février 2022. URL : http:// journals.openedition.org/encyclopedieberbere/676 ; DOI : https://doi.org/10.4000/ encyclopedieberbere.676

Ce document a été généré automatiquement le 17 février 2022.

(c) Tous droits réservés 


\section{Mzab (ou M’zab) : Histoire et société}

\section{S. Doumane}

\section{Données physiques et climatiques}

1 Le Mzab est une vallée de presque $40.000 \mathrm{~km}^{2}$ configurée par l'oued Mzab et ses affluents, située à la limite du Bas-Sahara algérien, entre les oasis de Laghouat au nord et de Ouargla au sud. La structure géomorphologique dans laquelle s'est constituée cette vallée est un plateau rocheux, façonné par l'érosion depuis l'ère quaternaire, d'altitude variant entre $300 \mathrm{~m}$ et $800 \mathrm{~m}$, appelé hamada par les nomades, terme repris par les géographes et les ethnologues français du 19 siècle.

2 Aride, son climat se distingue par une période chaude et sèche allant d'avril à octobre et des précipitations faibles et irrégulières le reste de l'année ( $60 \mathrm{~mm} / \mathrm{an}$ en moyenne). La température peut dépasser les 45 degrés en été (juillet-août) et descendre jusqu'à 5 degrés en hiver (janvier). L'amplitude thermique est très élevée, atteignant un taux moyen de 35 degrés.

3 Grâce à la présence d'une nappe albienne d'une profondeur de 100 à 400 mètres s'étendant sur une cinquantaine de millions d'hectares, les hommes y ont développé depuis des siècles une pratique agricole oasienne dite à étages ou strates, où le palmier dattier occupe la strate supérieure, l'arbre fruitier la strate intermédiaire et les cultures maraîchères et fourragères la strate inférieure. C'est cette nappe géologique qui garantit la survie de l'oasis lors des périodes de sécheresse, la nappe phréatique (10 à 50 mètres) s'alimentant par infiltration des eaux de surface étant très dépendante des précipitations et des rares crues des oueds.

\section{Dénomination et peuplement}

4 Les sources arabes et ibadites du Moyen Age utilisent le mot Banu Masâab pour désigner une grande partie de la population installée dans la vallée du Mzab. Ibn Khaldoun dans l'histoire des Berbères, parle de groupes berbères apparentés aux Zénètes, qu'il dénomme Musâab (ou Mosâab). Plus récemment, l'auteur ibadite d'un essai 
d'histoire de la région (Amhammad Atfayyash 1914) recourt à l'étymologie d'un terme mecquois "Mizab» (gouttière) et appelle ses coreligionnaires Bani Mizab (fils de Mizab), s'ingéniant à établir une hypothétique origine mecquoise des ibadites ou du moins un lien sacré (le mot Mizab ne désignant pas une banale gouttière mais celle du sanctuaire de la principale mosquée de la Mecque).

Un anthropologue mozabite (Brahim Chérifi 2003), s'appuyant sur des documents d'histoire du peuplement de la vallée du Mzab et l'analyse étymologique du mot par lequel les Mozabites se nomment eux-mêmes, At Mzab (les gens du Mzab), propose une explication plus réaliste. Il s'agirait du syntagme berbère At n zab = « Les gens du Zab », légèrement altéré par la transformation de la préposition $n$ ("de », «appartenant à ») en $m$ (phénomène linguistique signalé dans différentes variantes de la langue berbère). Cette interprétation est corroborée, selon notre auteur, par des données historiques : le Zab (correspondant à l'actuel Ziban recouvrant le sud des Aurès et les environs de Biskra) est une des régions d'origine migratoire des populations ibadites du Mzab. En effet, il semble bien que la dénomination Mzab est liée à la migration ibadite la plus importante venant de l'est, l'Ifrikia et le Zab (Ibn Khaldoun, Histoire des Berbères, t.3) suite à l'offensive des tribus arabes hilaliennes dans la deuxième moitié du $\mathrm{XI}^{\mathrm{e}}$ siècle. Une autre source évoque cet exode des populations du Zab vers le Mzab sans établir la correspondance étymologique des deux mots (Huguet 1906) avant que la thèse de B. Chérifi n'apporte des éclairages nouveaux étayés par une documentation bilingue (arabe et français) et une bonne enquête de terrain facilitée par son immersion dans la société mozabite.

Ce dernier auteur avance, en effet, de nouveaux éléments de connaissance non seulement sur la signification du mot Mzab mais aussi et surtout sur l'origine et la diversité du peuplement mozabite.

7 La thèse communément admise en la matière est que la population ibadite établie dans le Mzab serait la descendante de celle en fuite de l'Etat Rostomide détruit en 909 par les Fatimides (Fekhar 1971), point de vue en vogue dans l'historiographie coloniale du XIX $^{\mathrm{e}}$ siècle (Masqueray 1879) et largement admise par les Mozabites eux-mêmes. En fait, le peuplement du Mzab s'est constitué aussi bien avant qu'après la chute de l'Etat Rostomide. Bien avant l'islamisation du Sahara et l'avènement de l'ibadisme, on signale des traces de vie humaine à différents endroits du désert nord-africain, notamment dans sa partie septentrionale (oasis de Ouargla, d'Arigh...). Dans la vallée du Mzab, des foyers néolithiques (Roffo 1934) et des gravures rupestres (Abonneau 1983) attestent d'une présence humaine remontant à quelques milliers d'années (au moins à 3000 ans avant J.C selon la dernière étude du CNRPAH, Alger, 2000). L'islamisation a commencé dans la seconde moitié du VIII siècle (Lewicki 1970) et s'est rapidement généralisé, notamment dans sa variante kharédjite (sufrite et ibadite). C'était le fait, pour l'essentiel, des Berbères qui trouvèrent dans ce schisme de l'Islam, une doctrine plus conforme à leurs mœurs (cf. Ibadites, EB XXIII), ils parvinrent à fonder de petits Etats dont les plus importants étaient celui de Tripoli à l'est du Maghreb en 743 (par les Zénètes de Tripolitaine) et, surtout, celui de Tihert à l'ouest vers la fin des années 770 (par les Rostomides). La chute de l'Etat rostomide en 909 sous les coups de boutoir du chef fatimide Abu Abdallah Al-Shî'i mit fin définitivement à l'implantation des Ibadites qui virent depuis leurs espaces se rétrécir petit à petit et ne purent se maintenir que dans quelques micro-territoires isolés (île de Djerba, montagne du Nefusa et surtout dans l'oasis du Mzab). Tout laisse penser que la fin de l'expérience ibadite de Tihert a 
ébranlé non seulement les habitants de cette cité mais aussi l'ensemble des communautés ibadites d'Afrique du Nord, ce qui les aurait pousser à converger vers certaines oasis pour finir, enfin, dans la vallée du Mzab dont les premiers habitants se seraient convertis à l'ibadisme sous l'influence de missionnaires ibadites (Chérifi 2003). La vallée a accueilli diverses populations dont l'intégration a été facilitée par la proximité sociologique, linguistique et religieuse. Toutefois, à en croire certains auteurs (Ibn-Khaldoun, Huguet), les apports humains les plus importants sont dus à l'offensive des tribus arabes hilaliennes qui ont provoqué le déplacement des Ibadites $\mathrm{du}$ Maghreb oriental. Cette vague migratoire a marqué durablement l'imaginaire ibadite (Atfayyash, Risâla 1914). Les ibadites de Ouargla et de Sédrata qui étaient alors des cités florissantes grâce au commerce caravanier, ne rejoignirent le Mzab qu'au XIII ${ }^{e}$ siècle, fuyant à leur tour les persécutions des Almoravides. Au total, on peut penser que la succession de persécutions endurées par les Ibadites était de nature à renforcer chez eux la croyance en l'existence d'une oasis-refuge ou mythe de Gugraf (Lewicki 1970) qui les a sans doute encouragé a s'agglutiner dans la vallée du Mzab. On ne peut donc parler d'homogénéité ethnique mais plutôt d'une diversité des origines ; il $\mathrm{y}$ a, par contre, constitution au fil des siècles d'une communauté fondée sur une réelle endogamie et surtout une unicité religieuse (Chérifi 2003).

8 C'est à partir du XI ${ }^{e}$ siècle que les Ibadites ont commencé à s'agglomérer dans les sept cités (cinq: Ghardaïa, Beni Isguen, Melika, Bou Noura, El Atteuf, établies autour de l'oued Mzab qu'on appelle la Pentapole et deux: Guerrara et Berriane relativement excentrées par rapport à la Pentapole, à une centaine de kilomètres) qui constituent aujourd'hui encore l'essentiel du "pays» mozabite ((cf. "La cité mozabite », EB XIII). Jusque-là, la vallée du Mzab était parsemée d'un certain nombre de petits campements ou villages établis autour de points d'eau et selon un ordre lignager, qu'on appelait igherman et dont certaines ruines subsistent encore, telles Agherm $\mathrm{n}$ Wadday, Agnunnay, Agherm n Tlazdit... (Chérifi 2003).

9 La concentration de ces igherman est, sans doute, due à des raisons défensives puisque les nouvelles agglomérations se sont (re)constituées sur des buttes rocheuses surplombant la vallée. Leurs dates de fondation ne sont pas connues avec exactitude mais on estime que les cinq premières cités ont vu le jour au cours du $\mathrm{XI}^{\mathrm{e}}$ siècle; les dates retenues sont souvent différentes d'une source à l'autre, en raison probablement $\mathrm{du}$ référent fondateur pris en compte dans le récit de fondation (le campement d'origine ou le nouveau site d'implantation?). C'est ainsi par exemple que la date de création de Beni Isguen varie, selon les sources, $\mathrm{du} \mathrm{XI}^{\mathrm{e}}$ au XII ${ }^{\mathrm{e}}$ siècle voire XIII ${ }^{\mathrm{e}}$ siècle (Atfayyash 1914, Chérifi 2003). Quant aux deux cités mozabites de création plus récente, elles datent du XVII siècle et sont constituées de lignages ibadites, détachés de leurs coreligionnaires pour des raisons de conflits internes. A Guerrara er Berrianne, les Ibadites sont moins nombreux que dans les autres cités du Mzab.

\section{L'habitat}

10 La cité mozabite (cf. EB XIII) s'est originellement constituée autour d'une mosquée qu'entourent les premières habitations formant un premier quartier. A l'arrivée de nouvelles recrues et/ou de la croissance démographique des autochtones nécessitant la construction de nouvelles habitations, vont se greffer d'autres quartiers selon une configuration concentrique. La structure architecturale en formation épouse le relief 
du lieu et évolue de haut en bas, les ruelles convergeant vers le marché situé en dehors des remparts. Ces derniers enfermant la cité sont susceptibles d'être poussés plus loin au fur et à mesure que la cité s'agrandit. Tandis que la mosquée érigée généralement sur un ancien site cultuel (dit â̂ssas, gardien, en berbère) est le lieu sacré par excellence, le marché situé en dehors des remparts est un lieu profane où s'exercent les échanges économiques, les contacts avec les étrangers et autres activités.

11 A l'origine, la cité mozabite est conçue et bâtie dans une optique défensive. Les Mozabites aisés détiennent dans les palmeraies des alentours des maisons de villégiature où ils se réfugient lors des grandes chaleurs d'été.

\section{La cohabitation avec les Arabes malékites}

Malgré l'étendue de l'espace saharien, les rapports entre les oasiens sédentaires et les nomades ont toujours été denses, évoluant entre coopération et conflit. En devenant un important relais du commerce transsaharien à partir du XII ${ }^{\mathrm{e}}$-XIII ${ }^{\mathrm{e}}$ siècle (suite au déclin des cités voisines, Ouargla et Sédrata), les Mozabites ont du recourir aux services de certaines tribus nomades. Ces nomades étaient engagés comme convoyeurs et protecteurs de caravanes et s'insinuèrent par la suite dans les rapports inter-clans mozabites comme guerriers; ils se rendirent ainsi nécessaires dans les équilibres entre les soffs* ibadites rivaux. Trois grandes tribus nomades, les Béni Merzoug, les M'dabih et les Chaâmbas, toutes de rite malékite campèrent d'abord à une certaine distance des cités mozabites avant de s'en approcher et s'installer à l'intérieur même de quelques quartiers ibadites. Ce faisant, ils se sédentarisent, acquièrent des biens, notamment des habitations, et, ne jouant plus les anciens rôles qui leur étaient assignés, leur présence devient de plus en plus problématique. En fait, la coexistence ente Ibadites et Malékites a toujours été émaillée de frictions sinon de conflits ouverts avec, parfois violence ; les affrontements récurrents de ces dernières années ne sont que le prolongement paroxystique d'une cohabitation difficile.

Depuis la constitution de leurs cités dans les oasis du Mzab, les Ibadites ont toujours essayé de réglementer la présence des étrangers dans leur espace de vie, notamment celle de leurs voisins nomades. Au cours du XVIII ${ }^{\mathrm{e}}$ siècle, diverses délibérations de leurs djemaâs* ont édicté un certain nombre de mesures restrictives destinées à limiter la présence et l'influence de ces nomades. De celle de la djemaâ de Ghardaïa qui tendait à limiter le nombre d'habitations des M'dabih (début du XVIII ${ }^{\mathrm{e}}$ siècle) à celle prise à un niveau confédéral qui interdisait la vente de maisons aux nomades (fin du XVIII ${ }^{\mathrm{e}}$ ), en passant par la mesure d'exhérédation de leurs descendants (milieu du XVIII siècle), toutes ces mesures montrent à quel point les Mozabites sont obnubilés par la cohésion de leur communauté. D'autres mesures tout aussi dissuasives seront prises au cours du XIX siècle, ce qui n'empêcha pas des conflits violents entre Ibadites et Malékites mais aussi entre segments ou soffs ibadites.

14 Aujourd'hui, si le nombre d'anciens nomades semble diminuer à l'intérieur des cités mozabites, elle se renforce considérablement dans l'espace inter-cités et dans l'ensemble de la vallée, à la faveur de l'insertion de plus en plus poussée du Mzab dans les rouages de l'administration de l'Etat central (le Mzab est devenue Wilaya de Ghardaïa en 1984) et du dynamisme économique du Mzab (appel de main d'œuvre, proximité des zones pétrolifère et gazière, sédentarisation des nomades...). On assiste de plus en plus à un déséquilibre démographique en défaveur des Ibadites, ce qui rend 
les tensions entre communautés encore plus vives, aggravée par l'installation anarchique des nouveaux venus : occupation des berges et du lit de l'oued, urbanisation désordonnée, constructions inadaptées (Chérifi 2003).

\section{L'ibadisme et les pratiques religieuses}

Les Ibadites cultivent un particularisme religieux dont les racines remontent au différent qui sépara le Khalife Ali de certains de ses partisans, suite au compromis qu'il négocia avec le gouverneur de Syrie, Moawiyya (Cf. Ibadites, EB XXIII).

A leur début, ils fondèrent des petits Etats ou Imamats, d'abord au Moyen-Orient (Yémen, Irak...) puis en Afrique du Nord pour perpétuer, au besoin par la force, leur doctrine. C'est ainsi qu'est née la Halqa, institution autant guerrière que religieuse, destinée à renforcer les assises de ces petites entités étatiques. Plus tard, suite à l'effondrement du dernier imamat, celui de Tihert, cette institution s'est peu à peu transformée en simple méthode d'enseignement et de perpétuation idéologique de la doctrine ibadite ; la Ḥalqa prend alors la forme d'une assemblée circulaire d'apprenants autour d'un maître. N'y accèdent que les postulants qui passent le cap d'une période probatoire pendant laquelle ils sont soumis à des épreuves sévères faites de vie ascétique, d'isolement et de rigueur physique et morale. Certaines Halqa acquièrent de la réputation et s'érigent en écoles.

Jusqu'à la fin du XIII ${ }^{\mathrm{e}}$ siècle, le Mzab n'était pas encore un lieu de rayonnement de l'ibadisme, contrairement à la cité voisine de Ouargla et à celles plus lointaines de Djerba, de Nefusa... Mais la nécessité s'y faisait sentir, en raison de l'importance que prenaient les cités du Mzab tant au plan démographique (afflux de population) qu'économique (développement d'activités commerciales). C'est ainsi qu'au début du XIVe siècle apparait le premier texte fondateur de la Ḥalqa du Mzab (Siyyâr 'Azzaba Ghardaïa) dont l'objectif est de former l'élite ibadite du Mzab (Iâzzaben); elle allait vite s'imposer et se perfectionner. Au cours du $\mathrm{XV}^{\mathrm{e}}$ siècle, une réforme est intervenue, instituant un enseignement à deux niveaux : Le premier composé des Imessurda (dérivé nominal, à préfixe mes-, de urdu, "texte liturgique »), apprenants dont la fonction est de réciter le Coran et les autres textes religieux et le second, les Irwen (de ari ou aru : écrire) formé d'étudiants de niveau supérieur, destinés à devenir l'élite savante d'où émane l'autorité politique et religieuse.

Ce renouveau religieux connaît son apogée au XVIII ${ }^{\mathrm{e}}$ siècle, détrônant les autres centres maghrébins, avant de connaître un certain déclin dans la deuxième moitié du $\mathrm{XIX}^{\mathrm{e}}$ siècle. Il semble que ce relatif déclin résulte de l'influence coloniale française qui introduisit l'école laïque dont laquelle s'engouffra la bourgeoisie commerçante mozabite. En réaction, l'institution religieuse se cantonna dans des attitudes conservatrices (surtout à Beni Isguen) peu en phase avec les exigences de la nouvelle donne sociale. Un mouvement réformateur, sous la houlette de Brahim Bayoud de retour d'un séjour d'études à Tunis et au Caire au début du $\mathrm{XX}^{\mathrm{e}}$ siècle, tente d'apporter quelques innovations à même d'ouvrir la Mzab aux mutations en cours (adoption de certaines techniques, abandon de l'interdiction faite aux femmes de sortir du Mzab, réforme de l'enseignement...). En fait, l'action de Brahim Bayoud et de son courant réformateur s'inscrivait dans la mouvance réformiste des Ulémas algériens et de leur chef de file Ben Badis; ce qui, aux yeux des autres religieux dits conservateurs, constituait un danger pour la doctrine ibadite. 

doctrine ibadite originelle; la différence entre les deux courants réside surtout dans le rôle qu'ils assignent au dogme religieux : les conservateurs sont (paradoxalement?) pour le maintien d'une certaine distinction entre le sacré et le profane tandis que les réformateurs militent en faveur de l'amalgame entre vie civile et vie religieuse. A l'heure où l'Islam politique bouleverse la sociologie religieuse en Algérie et dans l'ensemble du Maghreb, ce clivage doctrinal qui traverse la société mozabite mérite d'être suivi de près.

\section{L'activité économique}

Le dynamisme économique des Ibadites du Mzab est proverbial. Il est fondé sur une ancienne tradition commerciale dont les débuts se confondent avec la constitution des cités mozabites à partir du XI ${ }^{\mathrm{e}}$ siècle. En effet, l'essor du Mzab est liée historiquement au développement de l'activité commerciale dont le Sahara fut le théâtre pendant des siècles, depuis le Moyen-Âge jusqu'à la pénétration coloniale française. Contrairement à une idée reçue, ce dynamisme s'explique moins par les conditions objectives dans lesquelles sont nées les cités mozabites (hostilité du milieu physique, isolement et éloignement des bordures de la Méditerranée et des grandes villes du Nord...), que par un concours de circonstances historiques, favorisées il est vrai par la culture ibadite (culte du travail, solidarité lignagère, sobriété et propension à l'accumulation). L'avènement de la prospérité commerciale du Mzab est, en effet, liée aux péripéties de l'histoire politique et économique de l'Afrique du Nord en général et du Sahara en particulier dont les Mozabites ont incontestablement tiré profit grâce aussi à leur habileté et leur savoir-faire.

21 La cause inaugurale, si l'on peut dire, est le déclin ou la ruine de la cité-carrefour du commerce caravanier au XI ${ }^{e}$ siècle, Ouargla, qui fut au cœur d'un réseau d'échanges qui va de l'Afrique subsaharienne à la Méditerranée, avec des ramifications à l'ouest et à l'est (T. Lewicki, Ibn-Khaldoun). L'axe Ouargla-Kairouan fut particulièrement florissant avant qu'il ne tombe en ruine sous la pression hilalienne à partir de 1057 (prise de Kairouan).

22 La ruine de Ouargla (attaquée de nouveau par les Hammadites en 1067 puis par les Almoravides en 1087) profita directement au Mzab voisin qui sut prendre place dans le circuit du commerce caravanier. Opportunistes à souhait, les Ibadites du Mzab n'en restèrent pas là ; ils s'adaptèrent judicieusement aux évolutions ultérieures de l'histoire de la région.

Avec les Ottomans qui prirent le contrôle de la côte méditerranéenne et des grandes cités (Alger, Constantine...) dans la deuxième moitié du XVI ${ }^{\mathrm{e}}$ siècle, ils nouèrent des relations avantageuses pour leurs commerces. C'est ainsi que les marchands mozabites organisés en corporation à Alger, bénéficièrent du monopole de certaines activités : abattoirs, moulins à farine, bains... Les territoires sous contrôle ottoman s'ouvrirent à leurs activités qui se redéploient, désormais vers le Nord, atteignant la Tunisie et même la côte atlantique. En échange, ils fournissent maints services au Dey, lui accordent des prêts et l'informent sur la situation des tribus se trouvant sur la route du Mzab. Ainsi, les Mozabites établissent leur négoce dans le Nord, allant jusqu'à installer leurs comptoirs en Tunisie et la côte atlantique sous contrôle portugais et le Mzab renforce sa position de plaque tournante entre le Nord et le Sud.

Encyclopédie berbère, 32 | 2010 

régions désertiques avant de s'y atteler dans les années 1850. Les Mozabites surent une nouvelle fois sauvegarder leurs intérêts en négociant avec le maréchal Randon un statut d'autonomie, moyennant un certain nombre de concessions dont le paiement d'un tribut annuel et d'un impôt sur les marchandises importées. Ils purent se faire une place dans la stratégie coloniale de contrôle du commerce transsaharien dans laquelle les populations sahariennes (Mozabites, Chaâmbas, Touaregs) furent conviées à jouer un rôle. Dans le même temps, grâce à l'ouverture de routes, les commerçants mozabites multiplièrent leurs implantations dans les villes $d u$ Nord et adaptèrent leurs commerces à leurs clientèles (musulmanes, européennes, rurales, urbaines). L'extinction du commerce saharien après la seconde guerre mondiale sous le poids de la concurrence des produits français amoindrit sensiblement la place du Mzab, mais les Mozabites trouvèrent la parade en tournant leurs activités vers le Nord de l'Algérie et même la France métropolitaine.

Après l'indépendance de l'Algérie, l'exploitation des hydrocarbures allait donner un coup de fouet inattendu à l'activité économique du Mzab. Situé sur l'axe routier conduisant aux champs pétrolifères et gaziers (Hassi R'mel et Hassi Messaoud) les cités mozabites ne peuvent que tirer profit des activités induites par l'acheminement des équipements et des divers approvisionnements que nécessite le fonctionnement de ces champs. Mais le grand boom de l'économie de la vallée du Mzab est consécutif aux options politiques algériennes des années 60-70 (étatisation) et industrielles (industrie lourde) qui ont laissé des opportunités d'investissement insoupçonnées aux capitaux mozabites dans la petite entreprise et l'industrie légère. Heureuses de voir s'ouvrir un marché (les bases pétrolières et gazières) à proximité, les entreprises mozabites redoublèrent d'efforts pour répondre à une demande en plein boom de certains produits de consommation courante (agro-alimentaire, petit outillage, entretien...). La flambée du prix du pétrole au début des années 1970 déclenche une forte demande d'investissements au point où les autorités mozabites lancèrent des mots d'ordre pour investir au "pays", rapatrier des capitaux et inciter leurs coreligionnaires à ne pas s'expatrier (Chérifi 2003). Une aubaine que les Mozabites ne laissèrent pas s'échapper d'autant que la pénurie des biens de consommation rendait le marché insatiable. Aussi, les zones industrielles du Mzab, notamment celle de Ghardaïa et de Guerrara, connurent un essor digne de pays développés. On produisait de tout: industrie mécanique, électrique, plastique, chimique, alimentaire etc...Et la vallée du Mzab devint une fourmilière où se bousculent hommes d'affaires, ouvriers, vendeurs, acheteurs... Elle étouffe, de ce fait, sous le poids d'une urbanisation sauvage, d'un surpeuplement et d'une dégradation accélérée de l'environnement oasien. En quelque sorte, la rançon du succès.

Aujourd'hui, l'ouverture des frontières aux marchandises internationales met à mal l'industrie du Mzab. On y assiste, selon l'Association des Industriels du Mzab (enquête de $\mathrm{B}$. Chérifi) à une attitude prudente et attentiste des entrepreneurs mozabites, en quête de nouvelles opportunités. On se rabat vers le commerce que les capitaux familiaux n'ont pas déserté, l'épargne, l'investissement foncier, les cliniques médicales et certaines filières agro-alimentaires (lait et produits dérivés). Mais de l'avis général, le Mzab a supporté la crise politico-économique des années 1990 et début des années 2000 mieux que les autres régions d'Algérie. C'est dire que les capacités d'adaptation des Ibadites d'Algérie sont toujours en éveil. 


\section{BIBLIOGRAPHIE}

ABONNEAU J., 1983 - Préhistoire du Mzab, wilaya de Laghouat, Algérie, thèse de $3^{\mathrm{e}}$ cycle art et archéologie, Paris I-Sorbonne.

AмAт Ch., 1888 - Le Mzab et les Mzabites, Challamel et C ${ }^{\text {ie }}$ Editeurs, Paris.

AUCAPITAINE H., 1867 - Les Béni Mézab, Sahara algérien, Challamel, Paris.

BOUSQUET Chr., 1986 - «L'habitat mozabite au Mzab », Annuaire de l'Afrique du Nord, T. XXV.

AUMASSIP G., FERHAT N., MOSTÉFAOUI O., 2000 - Rapport de mission, Ghardaïa, CNRPAH, Alger.

CARRET J., 1960 - « Le particularisme ibadite au Mzab », L'Afrique et l'Asie, 49.

CHÉRIFI B., 2003 - Etudes d'anthropologie historique et culturelle sur le Mzab, thèse de doctorat d'anthroplogie, Université de Paris VIII-Saint-Denis.

COYNE A., 1879 - le Mzab, Jourdan, Alger

DELHEURE J., 1986 - Faits et dires du Mzab, SELAF (Peeters), Paris.

FEKHAR B. 1971 - Les communautés ibadites en Afrique du Nord depuis les Fatimides, thèse pour le doctorat d'Etat d'histoire Paris I-Sorbonne.

GROSSMAN Cl., 1976 - Aperçu sur l'histoire religieuse des Ibadites, thèse de $3^{\mathrm{e}}$ cycle, Paris I-Sorbonne.

HUGUET J., 1903 - « Origines et migrations des tribus berbères et particulièrement des Beni-Mzab (1906) », Revue de l'Ecole d'Anthropologie de Paris, t. XVI.

IBN KHALDOUN Abderrahmane, 1999 - Histoire des Berbère, $2^{\mathrm{e}}$ édition, Geuthner, Paris.

LEPES R., 1925 - «Quelques documents sur la corporation des Mozabites d'Alger dans les premiers temps de la conquête (1830-1838)», Revue Africaine, p. 323-324.

LEWICKI T., 1970 - « Une croyance des Ibadites nord-africains sur la fin du monde, le pays de Gugraf », Actes du $V^{\text {ème }}$ congrès mondial des arabisants et des islamisants, Bruxelles.

LEWICKI T., 1973 - Etudes maghrébines et soudanaises, Académie polonaises des sciences, comité des études orientales, Varsovie.

MASQUERAY E., 1886 - Formation des cités chez les populations sédentaires en Algérie, Kabyles du Djurdjura, Chaouia de l'Auras, Beni Mezab, Leroux, Paris. Réédition (Préface de Colonna, Fanny) Aixen-Provence, Edisud, 1983.

MERCIER M., 1922 - La civilisation urbaine au Mzab, étude de sociologie africaine, Imprimerie Pfister, Alger.

MERGHOUB B., 1971 - Le développement politique en Algérie, étude des populations de la région du Mzab, Armand Colin, Paris.

ROFFO P., 1934 - Les civilisations paléolithiques du Mzab, Imprimerie Heintz, Alger. SHALER W., 1830 - Esquisse de l'Etat d'Alger, les Beni Mozab, Imprimerie GauthierLaguianie, Alger. 
INDEX

Mots-clés : Algérie, Economie, Géographie, Mzab, Peuplement, Population 